\title{
INFLUÊNCIA DOS FATORES BIOLÓGICOS E SOCIOECONÔMICOS NO DESENVOLVIMENTO NEUROPSICOMOTOR DE PRÉ-ESCOLARES
}

\section{Juliana Fernandes Pereira}

Fisioterapeuta pela Universidade Estadual de Goiás (UEG), Brasil.

\section{Cibelle Kayenne Martins Roberto Formiga}

Fisioterapeuta, Doutora em Ciências Médicas (Saúde Mental) pela FMRP/USP; Docente Doutora do Curso de Fisioterapia da Universidade Estadual de Goiás (UEG); Programa de Bolsa de Incentivo à Pesquisa e Produção Científica (PROBIP) da UEG, Brasil.

E-mail: cibellekayenne@gmail.com

\section{Martina Estevam Brom Vieira}

Fisioterapeuta, Doutora em Ciências Médicas (Saúde Mental) pela FMRP/USP; Docente Doutora do Curso de Fisioterapia da Universidade Estadual de Goiás (UEG), Brasil.

\section{Maria Beatriz Martins Linhares}

Psicóloga, Docente do Programa de Pós-graduação em Saúde Mental pela Faculdade de Medicina de Ribeirão Preto, Universidade de São Paulo (USP), Brasil.
RESUMO: O objetivo do estudo foi caracterizar as variáveis socioeconômicas e psicossociais de crianças que frequentam creches públicas e avaliar a influência destes fatores no desenvolvimento neuropsicomotor. Realizou-se um estudo analítico-observacional transversal com 61 crianças, de ambos os sexos, com idade de quatro a seis anos, frequentadoras das creches públicas na cidade de Goiânia (GO). O desenvolvimento infantil foi avaliado pelo teste de triagem de Denver II e foi aplicado questionário socioeconômico, classificação ABEP e questionários sobre os dados biológicos e clínicos da criança com os pais. Verificou-se que $77 \%$ das crianças avaliadas apresentaram risco na classificação global do teste de Denver II, sendo típicas nas áreas psicossocial, motor fino, motor amplo e linguagem. A análise da influência dos fatores revelou que a renda familiar (até $\mathrm{R} \$ 2.000,00$ ) esteve associada com maior porcentagem de risco no desenvolvimento da linguagem (64\%). Os demais fatores não tiveram diferença estatisticamente significativa. Conclui-se que crianças aparentemente normais possam apresentar risco em seu desenvolvimento e a necessidade de novos estudos que apontem a influência significativa dos fatores socioeconômicos e psicossociais sobre o desenvolvimento neuropsicomotor.

PALAVRAS-CHAVE: Desenvolvimento infantil. Pré-escolares. Creches. Fatores socioeconômicos.

\section{THE INFLUENCE OF BIOLOGICAL AND SOCIO- ECONOMIC FACTORS IN NEURO-PSYCHOMOTOR DEVELOPMENT OF KINDERGARTEN CHILDREN}

ABSTRACT: Socio-economic and psycho-social variables of children in government-run kindergartens are characterized and the influence of such factors on neuro-psychomotor development evaluated. A transversal analytic-observational study was undertaken with 61 children, males and females, aged $4-6$ years, in kindergartens in Goiânia GO Brazil. Child development was assessed by Denver Selection Test II and a socio-economic questionnaire, ADEP classification and questionnaires on the children's biological and clinical data were undertaken. Seventy-seven percent of children rank risk mark in global classification of Denver Test II, typically within the psychosocial area, fine motor, wide motor and language. Analysis for factor influence revealed that family income (up to $R \$ 2000$ ) was associated with greater risk percentage in language development (64\%). There was no statistical difference for the other factors. Although results revealed that apparently normal children showed development risk, further studies are required to weigh the significant influence of socio-economic and psycho-social factors on neuro-psychomotor development.

KEY WORDS: Child development; Kindergarten children; Creches; Socio-economic Factors. 


\section{INTRODUÇÃO}

O desenvolvimento neuropsicomotor é considerado um conjunto de alterações no comportamento da criança resultante da interação entre mudanças estruturais no corpo e influências ambientais, abrangendo quatro componentes principais: motricidade ampla, motricidade fina-adaptativa, linguagem e comportamento pessoal-social (SOUZA et al., 2008; BRITO et al., 2011).

A motricidade ampla se refere ao controle motor sobre a musculatura do eixo corporal (controle de cabeça, sentar, engatinhar e ficar de pé), a motricidade fina desenvolve-se a partir de movimentos generalizados que se tornam cada vez mais precisos, envolve a capacidade de segurar e manipular objetos (MATOS, 2009).

A aquisição e o desenvolvimento da linguagem resultam da interação entre as características individuais e ambientais, podendo ser quantitativa de acordo com o número de palavras que a criança pronuncia ao longo de seu desenvolvimento, e qualitativa em relação à elaboração de frases e o significado. A linguagem evolui paralelamente aos seus elementos como a articulação, o vocabulário, significado e semântica das palavras (MOUSINHO et al., 2008).

Segundo Cró e Pinho (2011), o comportamento pessoal-social está relacionado à maturação da personalidade e engloba a aprendizagem de valores, normas e regras, modo de pensar e agir, além da expressão e comunicação. O desenvolvimento do comportamento pessoal-social fundamenta-se na relação da criança com as outras pessoas que convive, gerando autocrítica de si mesma e pensamentos de responsabilidade.

O ambiente onde a criança vive ocasiona impacto tanto no crescimento quanto no desenvolvimento e diversos fatores podem influenciar a progressão do desenvolvimento normal de crianças (PILZ; SCHERMANN, 2007).

Estudos como Brito et al. (2011), Moraes et al., (2010), Souza et al. (2008) e Torquato et al. (2011) apontam a prevalência de atrasos no desenvolvimento neuropsicomotor relacionados a fatores socioeconômicos e psicossociais como escolaridade materna e renda familiar baixa, ausência do pai e depressão materna, e a fatores biológicos como o estado nutricional, idade gestacional e peso ao nascimento.

Segundo Biscegli et al. (2007), na fase préescolar, as instituições de educação infantil preconizam a promoção do desenvolvimento geral abrangendo os componentes físico, psicológico, intelectual e social, uma vez que os primeiros anos de vida são fundamentais para a aquisição de habilidades e conhecimentos específicos para esta faixa etária.

As creches ou pré-escolas são instituições que oferecem aos pré-escolares atividades educativas, estratégias de assistência e promoção de saúde com a finalidade de estimular o desenvolvimento e também servir de suporte para as famílias (YAMAMOTO et al, 2009).

Diante da prevalência de atraso no desenvolvimento de crianças que frequentam pré-escola, o objetivo geral do estudo foi verificar a influência dos fatores biológicos e socioeconômicos sobre o desenvolvimento neuropsicomotor de pré-escolares matriculados na rede municipal de ensino em GoiâniaGO.

De forma complementar, a pesquisa objetivou também caracterizar os indicadores biológicos e a história de saúde dos pré-escolares participantes da pesquisa; caracterizar os fatores socioeconômicos e psicossociais dos pré-escolares e suas respectivas famílias; analisar os quatro principais componentes (motricidade ampla, motricidade fina-adaptativa, linguagem e comportamento pessoal-social) das crianças em idade pré-escolar; verificar a influência dos fatores biológicos e socioeconômicos sobre o desenvolvimento neuropsicomotor global e nos quatro componentes avaliados das crianças em idade pré-escolar.

\section{METODOLOGIA}

O presente estudo teve um delineamento analítico-observacional transversal. Participou uma amostra de 61 crianças de ambos os gêneros em idade pré-escolar (04 a 06 anos) frequentadoras de Centros Municipais de Educação Infantil (CMEI) da cidade de Goiânia (GO).

Os critérios de inclusão do estudo foram: crianças 
de quatro a seis anos de idade cronológica; crianças que estavam devidamente matriculadas e que frequentavam o CMEI; as crianças cujos pais ou responsáveis autorizaram a participação no projeto por meio da assinatura do Termo de Consentimento Livre e Esclarecido.

Os critérios de exclusão do estudo foram os seguintes: crianças que apresentavam patologias infectocontagiosas; anomalias cromossômicas, patologias neurológicas, problemas ortopédicos e malformações congênitas; aquelas que se recusaram a participar da avaliação após pelo menos três tentativas dos examinadores em dias diferentes; aquelas que faltaram nos dias das avaliações;

Este estudo está previsto de acordo com as Diretrizes e Normas Regulamentadoras de Pesquisas envolvendo seres humanos (resolução 196/1996, do Conselho Nacional de Saúde). A presente pesquisa foi submetida e aprovada pelo Comitê de Ética em Pesquisa da Universidade Federal de Goiás no 195/2009, bem como teve autorização da Secretaria Municipal de Educação. Os pais e/ou responsáveis pelos bebês autorizaram a participação das crianças assinando o Termo de Consentimento Livre e Esclarecido.

A coleta de dados foi realizada em uma sala que os CMEIs disponibilizaram e, posteriormente, a coleta de dados com os pais ou responsáveis foi realizada no próprio CMEI ou na residência das famílias após agendamento prévio e segundo a disponibilidade destes. A fase de análise dos dados foi realizada no Núcleo Interdisciplinar de Pesquisa da Eseffego (Nipe), da Universidade Estadual de Goiás.

Para a realização do registro dos dados coletados foram utilizados os seguintes materiais e instrumentos:

a) Fichas de registro do Teste de Triagem do Desenvolvimento de Denver II (FRANKENBURG et al., 1992) adaptado para a língua portuguesa: $\mathrm{O}$ teste classifica a criança dicotomicamente em suspeita (risco) de atraso no desenvolvimento neuropsicomotor ou normal. Pode ser realizado em bebês desde o nascimento até seis anos de idade e avalia quatro aspectos do desenvolvimento da criança: relacionamento pessoal-social, linguagem, motor fino e motor amplo.

b) Questionário socioeconômico: questionário elaborado para a presente pesquisa, aplicado aos pais e/ou responsáveis para obter informações quanto à escolaridade paterna e materna, renda familiar, tipo de residência onde vive a criança, plano de saúde, dentre outras informações.

c) Critério da Classificação Econômica da Associação Brasileira de Empresas e Pesquisa (ABEP, 2009): foi administrado por meio de entrevista com os pais e/ou responsáveis, permitindo a classificação das famílias em classe A1, A2, B1, B2, C, D ou E. Sendo a classe A1 com melhores condições socioeconômicas (maior pontuação) e a classe $\mathrm{E}$ com as piores condições (menor pontuação). Essa escala varia de 0 a 34 pontos e avalia o poder de compra e escolaridade do chefe da família.

d) Roteiro de Caracterização Biológica da Criança em Idade Pré-escolar: foi aplicado por meio de entrevistas com os pais e/ou responsáveis, em que foram coletadas informações sobre a história clínica neonatal (idade gestacional de acordo com o Cartão de Saúde da Criança ou outro documento médico e informações sobre os períodos pré, peri e pós-natal) e a história das condições de saúde da criança (peso corporal, altura, história de doenças, internações, cirurgias, acidentes, uso de próteses, uso de medicamentos etc.) e sobre o relacionamento interpessoal (se a criança tem amigos, preferências de brincadeiras). Esse roteiro é uma adaptação do "Roteiro de Entrevista com as mães" do estudo de Carvalho, Linhares e Martinez (2001) e do "Roteiro de Caracterização dos Dados Biológicos" da tese de doutorado de Formiga (2009).

Os seguintes equipamentos foram utilizados para a coleta dos dados: brinquedos diversos, um lápis vermelho, papel branco, fichas com desenhos de cruz e quadrado, fita métrica para medir a alturas das crianças, 
balança analógica para verificar o peso corporal, ficha de registro dos instrumentos que serão utilizados, pranchetas, canetas e régua para anotar os dados coletados.

\subsection{PROCEDIMENTOS}

Foi solicitada a autorização da Secretaria Municipal de Educação para realizar o estudo nos CMEIs de Goiânia, os pesquisadores entraram em contato com o CMEI para informar sobre o estudo e marcar o horário das visitas. Os pais e responsáveis pelas crianças selecionadas foram esclarecidos sobre a pesquisa e seu caráter voluntário por meio de bilhetes anexados às agendas escolares de cada criança. Sendo que aqueles que concordaram com a participação da criança assinaram o Termo de Compromisso Livre e Esclarecido.

Foi realizada a avaliação da criança pelo teste de Denver II (FRANKENBURG et al., 1992), utilizando as subáreas motricidade ampla, motricidade finaadaptativa, linguagem e comportamento pessoal-social, por dois examinadores treinados, sendo que enquanto um examinador avaliava a criança o outro examinador marcava a ficha de registro do teste. O teste inclui vários itens que foram pontuados de acordo com a idade da criança com passou ("P") se a criança conseguiu realizar a tarefa ou falhou ("F") se não conseguiu realizar a tarefa. Essa avaliação foi feita em uma sala que o CMEI disponibilizou. As crianças foram avaliadas de acordo com a idade cronológica.

Após a avaliação, os pesquisadores entraram em contato com os pais ou responsáveis por cada criança para marcar a entrevista a fim de coletar dados sobre os aspectos socioeconômicos e de saúde da criança.

Foi realizada a organização de todos dados após a coleta por meio da conversão das fichas e resultados do teste em códigos na planilha do Excel. Com o banco de dados organizado foram selecionadas as variáveis para análise das variáveis socioeconômicas e psicossociais com base na estatística descritiva (média, desvio-padrão, frequência e porcentagens). As variáveis socioeconômicas selecionadas para a análise foram: renda familiar mensal, plano de saúde e classe econômica ABEP. As variáveis biológicas selecionadas para análise foram idade gestacional, $\mathrm{n}^{\mathrm{o}}$ de consultas no pré-natal, tipo de parto, peso ao nascer, altura ao nascer, ápgar no quinto minuto, altura da criança, peso e IMC da criança.

A partir da análise descritiva foram selecionados grupos de variáveis para a realização dos testes de comparação de grupos. Foi utilizado o teste Quiquadrado para as comparações das variáveis categóricas. As variáveis de desfecho foram os resultados das crianças no teste de Denver II considerando o desempenho global no teste e nos quatro componentes avaliados. Foi utilizado o programa estatístico SPSS, versão 22.0, para as análises dos dados. Foi adotado o nível de significância de $5 \%(p \leq 0,005)$ em todas as análises.

\section{RESULTADOS}

Na Tabela 1 são apresentadas as características biológicas e história de saúde das crianças da amostra do estudo ( $n=61)$. Verifica-se na Tabela 1 que prevaleceu na amostra crianças a termo, mães com seis ou mais consultas no pré-natal, tipo de parto cesárea, crianças com peso e altura adequadas ao nascimento.

Tabela 1. Dados biológicos e clínicos da fase pré-escolar $(n=61)$

(continua)

\begin{tabular}{cl}
\hline Indicadores biológicos e clínicos & Valores \\
\hline Idade gestacional - $\mathrm{f}(\%)$ & \\
\hline Pré-termo & $5(8)$ \\
A termo & $56(92)$ \\
\hline
\end{tabular}

$\mathrm{N}^{\circ}$ de consultas no pré-natal - $\mathrm{f}(\%)$

\begin{tabular}{ll} 
Mãe com $<6$ consultas & $5(8)$ \\
Mãe com $>$ ou $=6$ consultas & $55(92)$ \\
\hline
\end{tabular}

Tipo de parto - f (\%)

$\begin{array}{ll}\text { Normal } & 21(34) \\ \text { Cesárea } & 40(66)\end{array}$

Peso ao nascer

$\begin{array}{ll}\text { Média (DP) } & 3276(681) \\ \text { Mínimo - Máximo } & 460 \text { - } 4700\end{array}$

\begin{tabular}{ll}
\hline Altura ao nascer & \\
\hline Média (DP) & $48,7(4,2)$ \\
Mínimo - Máximo & 30 - 56 \\
\hline
\end{tabular}

Apgar no $5^{\circ}$ minuto 
(conclusão)

\begin{tabular}{cl}
\hline Indicadores biológicos e clínicos & Valores \\
\hline Média (DP) & $9,2(1,3)$ \\
Mínimo - Máximo & 5 - 10 \\
\hline Altura da criança & $117(4,9)$ \\
\hline Média (DP) & 107 - 130 \\
Mínimo - Máximo & \\
\hline Peso da criança & $21,3(4,5)$ \\
\hline Média (DP) & $15-47$ \\
Mínimo - Máximo & \\
\hline IMC da criança- f (\%) & $4(7)$ \\
\hline Baixo peso & $48(80)$ \\
Peso saudável & $4(7)$ \\
Risco de sobrepeso & $4(7)$ \\
Sobrepeso &
\end{tabular}

Nota: $\mathrm{f}$ - frequência; \% - porcentagem; DP - Desvio padrão;

$\mathrm{Na}$ Tabela 2 são apresentados os fatores socioeconômicos e psicossociais das famílias das crianças $(n=61)$.

Tabela 2. Dados socioeconômicos e psicossociais $(n=61)$

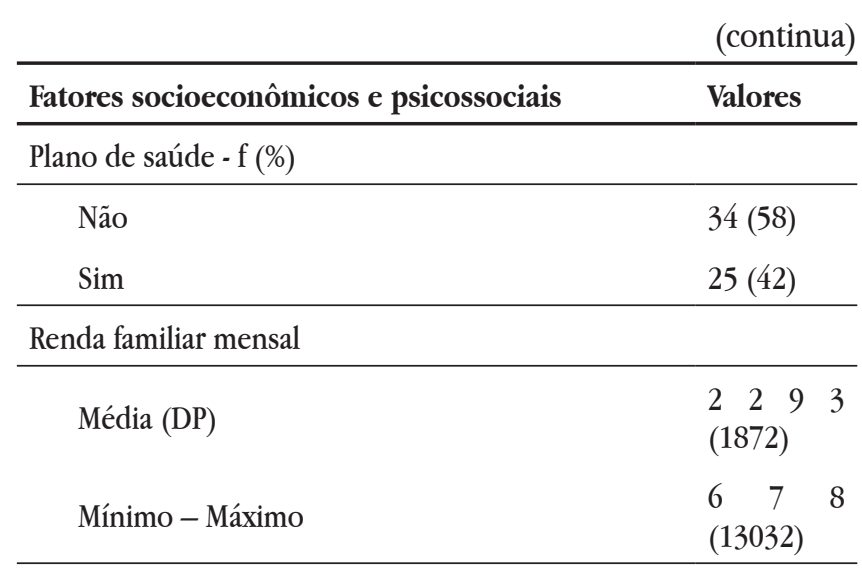

\begin{tabular}{ll}
\hline $\mathrm{N}^{\circ}$ de pessoas que moram com a criança & \\
\hline Média (DP) & $4(1)$ \\
Mínimo - Máximo & $2(7)$ \\
\hline
\end{tabular}

Classe econômica ABEP - $\mathrm{f}(\%)$

Classe E até C1 39 (64)

Classe $\mathrm{B} 2$ até $\mathrm{A} 1$

\begin{tabular}{ll}
\hline Idade da mãe & \\
\hline Média (DP) & $32(6)$ \\
Mínimo - Máximo & 19 - 49 \\
\hline Escolaridade da mãe - f (\%)
\end{tabular}

\begin{tabular}{|c|c|}
\hline & (conclus \\
\hline Fatores socioeconômicos e psicossociais & Valores \\
\hline Ensino fundamental & $22(37)$ \\
\hline Ensino médio ou superior & $37(63)$ \\
\hline \multicolumn{2}{|l|}{ Escolaridade do pai - f (\%) } \\
\hline Ensino fundamental & $26(46)$ \\
\hline Ensino médio ou superior & $31(54)$ \\
\hline \multicolumn{2}{|l|}{ Ocupação da mãe - f (\%) } \\
\hline Dentro do lar & $6(10)$ \\
\hline Fora do lar & $51(90)$ \\
\hline \multicolumn{2}{|l|}{ Estado civil dos pais - $\mathrm{f}(\%)$} \\
\hline União estável & $32(54)$ \\
\hline União instável & $27(46)$ \\
\hline \multicolumn{2}{|l|}{ Planejamento da gravidez - f (\%) } \\
\hline Não & $43(70)$ \\
\hline Sim & $18(30)$ \\
\hline \multicolumn{2}{|l|}{ Sexo da criança - $\mathrm{f}(\%)$} \\
\hline Masculino & $31(51)$ \\
\hline Feminino & $30(49)$ \\
\hline \multicolumn{2}{|l|}{ Cor da criança - $\mathrm{f}(\%)$} \\
\hline Branca & $19(31)$ \\
\hline Parda & $36(59)$ \\
\hline Negra & $6(10)$ \\
\hline \multicolumn{2}{|c|}{ Se a criança foi amamentada no seio da mãe - $\mathrm{f}(\%)$} \\
\hline Não & $3(5)$ \\
\hline Sim & $58(95)$ \\
\hline
\end{tabular}

Nota: f - frequência; \% - porcentagem; DP - Desvio padrão.

Quanto aos dados socioeconômicos apresentados na Tabela 2 , verifica-se que $58 \%$ das famílias não possuíam plano de saúde, $54 \%$ dos pais das crianças encontravamse em união estável, 63\% das mães apresentaram nível médio ou superior de escolaridade em relação aos pais que apresentaram apenas 54\%, 90\% das mães trabalhavam fora do lar. A classe econômica prevalente na amostra foram as classes $\mathrm{E}$ até $\mathrm{C} 1$, segundo o critério da ABEP.

Em relação aos dados psicossociais, verificase que a média da idade das mães foi 32 anos, 43\% das famílias não planejaram a gravidez de seus filhos e apenas 5\% não amamentaram, o sexo predominante foi 0 masculino e a cor parda mais prevalente.

Na Tabela 3 é apresentada a classificação da 
criança no teste de triagem de Denver II.

Tabela 3. Avaliação das Crianças pelo Teste de Denver II

\begin{tabular}{lc}
\hline Classificação da criança aos 5-6 anos & Valores \\
\hline Pessoal-social - $\mathrm{f}(\%)$ & \\
\hline Risco & $20(33)$ \\
Normal ou "não risco" & $41(67)$ \\
\hline Motor fino - f (\%) & $29(48)$ \\
\hline Risco & $32(52)$ \\
\hline Normal ou "não risco" & \\
\hline Linguagem - f (\%) & $27(44)$ \\
\hline Risco & $34(56)$ \\
\hline Normal ou "não risco" & $11(18)$ \\
\hline Motor amplo - f (\%) & $50(82)$ \\
\hline Risco & \\
\hline Normal ou "não risco" & $47(77)$ \\
\hline Total - f (\%) & $14(23)$ \\
\hline Risco
\end{tabular}

Nota: $\mathrm{f}$ - frequência; \% - porcentagem.

Verificou-se que $77 \%$ das crianças avaliadas apresentaram risco na classificação total do teste, sendo normais nas áreas pessoal-social, motor fino, motor amplo e linguagem.

$\mathrm{Na}$ Tabela 4 são apontadas as correlações entre os fatores biológicos e psicossociais sobre o desenvolvimento neuropsicomotor.

Tabela 4. Relação dos fatores biológicos sobre o desenvolvimento neuropsicomotor

\begin{tabular}{llcc}
\hline $\begin{array}{l}\text { Classificação } \\
\text { Denver }\end{array}$ & Variáveis & $\begin{array}{c}\text { Coeficiente de } \\
\text { Correlação (r)* }\end{array}$ & $\begin{array}{c}\text { Valor } \\
\text { de } \mathbf{p}\end{array}$ \\
\hline $\begin{array}{l}\text { Risco na } \\
\text { Linguagem }\end{array}$ & Parto normal & 0,32 & 0,010 \\
$\begin{array}{l}\text { Risco no Motor } \\
\text { Fino }\end{array}$ & $\begin{array}{l}\text { Menor altura ao } \\
\text { nascer }\end{array}$ & 0,39 & 0,007 \\
$\begin{array}{l}\text { Risco no Pessoal } \\
\text { Social }\end{array}$ & $\begin{array}{l}\text { Menor altura na } \\
\text { idade pré-escolar }\end{array}$ & 0,28 & 0,030 \\
\hline
\end{tabular}

*Teste de Correlação de Spearman
Verificou-se a correlação positiva entre o parto normal e risco na área da linguagem. A menor altura ao nascimento esteve associada ao risco na área motora fina. Crianças com menor altura na idade pré-escolar apresentaram mais risco na área pessoal-social.

Na Tabela 5 é apresentada a influência de fatores biológicos e socioeconômicos relacionados ao risco de atraso na área da linguagem.

Tabela 5. Influência dos fatores biológicos e socioeconômicos no desenvolvimento neuropsicomotor

\begin{tabular}{|c|c|c|c|c|c|}
\hline \multirow{2}{*}{$\begin{array}{l}\text { Fatores biológicos } \\
\text { e socioeconômicos } \\
\text { no desenvolvimento } \\
\text { neuropsicomotor } \\
\text { relacionados à } \\
\text { linguagem. }\end{array}$} & \multicolumn{2}{|c|}{ Normal } & \multicolumn{2}{|c|}{ Risco } & \multirow{2}{*}{$\begin{array}{c}\text { Valor } \\
\text { de } \\
\text { p }^{*}\end{array}$} \\
\hline & f & $\%$ & f & $\%$ & \\
\hline \multicolumn{6}{|l|}{ Renda familiar mensal } \\
\hline Até dois mil reais & 10 & 36 & 18 & 64 & \multirow{2}{*}{0,009} \\
\hline Acima de dois mil reais & 22 & 76 & 7 & 24 & \\
\hline \multicolumn{6}{|l|}{ Tipo de parto } \\
\hline Normal & 7 & 12 & 14 & 68 & \multirow{2}{*}{0,011} \\
\hline Cesárea & 27 & 67 & 13 & 33 & \\
\hline
\end{tabular}

Verificou associação entre risco na área da linguagem as crianças que nasceram de parto normal e pertencente às famílias com renda mensal de até $\mathrm{R} \$ 2.000,00$.

\section{DISCUSSÃO}

No presente estudo, foi verificada a associação de fatores socioeconômicos e psicossociais sobre o desenvolvimento neuropsicomotor de crianças matriculadas na educação infantil pública de Goiânia (GO) com idade entre quatro a seis anos. Além disso, foram avaliados os indicadores clínicos e de saúde da criança ao nascimento e na fase pré-escolar.

Em nosso estudo, a maioria das crianças avaliadas apresentou normais ou não apresentando risco no desenvolvimento nas áreas da linguagem, pessoal-social, motor amplo e motor fino, porém foram classificadas como "risco" no item classificação total do teste de Denver II. 
Os resultados encontrados são discordantes do estudo de Najjar et al. (2011) que verificaram a influência da condição socioeconômica sobre o desenvolvimento neuropsicomotor de 30 crianças com idade de zero a seis anos, de ambos os sexos, institucionalizadas em abrigos, foi encontrado que $94 \%$ das crianças apresentavam atrasos em todas as áreas do teste de Denver II.

No atual estudo, o parto normal foi considerado fator de risco para o desenvolvimento na área da linguagem (68\%), concordando com o estudo de Silva, Engstron e Miranda (2015), que avaliaram 112 crianças no Estado da Paraíba, onde se associou o parto vaginal com alteração no desenvolvimento neuropsicomotor com razão de chance (RC) de 4,4.

Ainda em relação ao parto, estudo realizado por Caldas et al. (2014) que avaliaram 77 crianças de dois a três anos de idade, prematuras e de baixo peso, na cidade de Cuiabá (MT), observou que crianças que nasceram de parto cesárea tiveram 44,6\% de alteração no teste de Denver II, com predomínio na área da linguagem, discordando do nosso estudo. Neste sentido, verifica-se que ainda não há consenso na literatura sobre as influências do tipo de parto no crescimento e desenvolvimento dos bebês.

No presente estudo, a baixa renda familiar correlacionou-se com o risco de atraso na área da linguagem, corroborando com o estudo de Costa, Cavalcante e Dell'Aglio (2015), em que foi avaliado o perfil de desenvolvimento da linguagem de 319 crianças com idade entre 36 a 48 meses na cidade de Belém (PA), e ocorreu 59,2\% de suspeita de atraso no desenvolvimento linguístico associado ao nível de pobreza das crianças.

No presente estudo, a menor altura ao nascimento esteve associada ao risco de atraso na área motora fina e a menor altura na idade pré-escolar com o risco na área pessoal-social. Tais resultados apontaram que o déficit no crescimento das crianças pode estar associado a dificuldades na área motora fina e na socialização, trazendo a importância das questões nutricionais tanto na gestação quanto nos primeiros anos de vida da criança.

A variável estatura está intimamente ligada à idade gestacional e ao peso da criança; estudos apontam que crianças prematuras e de baixo peso apresentam mais atraso no desempenho motor. Um estudo conduzido por Maggi et al. (2013) com 124 crianças de quatro anos de idade, distribuídas em dois grupos de acordo com a idade gestacional e peso ao nascimento, demonstrou que 53\% apresentaram atraso no desenvolvimento motor cognitivo e funcional em relação aos seus pares a termo.

Em nosso estudo $8 \%$ crianças pré-termo e o peso ao nascer em média de $3.276 \mathrm{~kg}$, sendo o menor peso 460 g e o maior peso $4.700 \mathrm{~g}$, não sendo encontradas relações significativas entre a idade gestacional e peso ao nascer com o resultado do desenvolvimento neuropsicomotor.

Crianças pré-termo apresentam desempenho motor, cognitivo e funcional desfavorável em relação a neonatos a termo em idade pré-escolar. Tais crianças exibem dificuldades no aprendizado, comportamento e na realização das atividades de vida diária, além de apresentarem menor rendimento escolar.

Outro fator biológico importante em relação ao desenvolvimento é o peso ao nascer. No estudo de Pulido et al. (2015) procurou-se estabelecer a relação entre peso ao nascer e a maturidade neuropsicológica em crianças pré-escolares na cidade de Tunja (Colômbia). Foram avaliadas 78 crianças com idade de 36 a 78 meses. Foram detectadas diferenças significativas entre peso ao nascer e o questionário de Maturidade Neuropsicológica (CUMANIN), sendo que as crianças do sexo masculino apresentaram pior desempenho.

Em nosso estudo, $80 \%$ das crianças apresentavam peso saudável de acordo com o Índice de Massa Corporal (IMC) na fase pré-escolar, sendo apenas 7\% com baixo peso e 7\% com sobrepeso. Loosli et al. (2012) relacionaram os dados do crescimento e desenvolvimento com o IMC em crianças institucionalizadas em creche. Foram avaliadas 30 crianças com idade cronológica entre dois a cinco anos, sendo aplicado o teste de triagem do desenvolvimento Denver II. A maioria das crianças estava com o peso adequado em todas as faixas etárias estudadas, observou-se que o valor do IMC/ idade não influenciou em nenhuma das áreas do teste de Denver II, concordando com nosso estudo, em que não foram encontradas diferenças significativas em IMC e desenvolvimento neuropsicomotor.

$\mathrm{Na}$ análise de associação dos fatores de risco, verificou-se que o planejamento da gravidez se 
correlacionou com a idade da mãe e o estado civil dos pais, em que a média de idade das mães foi de 32 anos e $54 \%$ dos pais encontravam-se em união estável.

Este resultado concorda com os encontrados no estudo de Tiago et al. (2015), em que das 66 crianças provenientes do Centro Educacional Infantil Sol Nascente na cidade de São Paulo, 50\% dos pais das crianças tinham idade entre $26-33$ anos, $40 \%$ eram casados, indicando que casais que vivem juntos esperam mais tempo para ter filhos.

Um fator que contribui para maior permanência das crianças nas creches é a ocupação da mãe. No atual estudo, $90 \%$ das mães trabalhavam fora do lar, o que resulta em menor estímulo materno oferecido à criança. Tal resultado concorda com o estudo de Silva, Engstron e Miranda (2015), em pesquisa realizada na Paraíba com 112 crianças e suas respectivas mães, em que 74,1 \% tinham ocupação fora do lar e foi observado alteração no desenvolvimento, sendo a área da linguagem mais afetada com $52,7 \%$ de atraso.

Nosso estudo correlacionou a ocupação da mãe com o nível de escolaridade das mães, em que $37 \%$ das mães apresentaram ensino médio ou superior, e com a média de dois filhos. Este resultado está de acordo com o resultado encontrado no estudo de Tiago et al. (2015) que identificaram os aspectos do ambiente familiar e a influência deste sobre o desenvolvimento de das crianças e encontrado média de filhos igual ou superior a dois filhos, o que resulta na dificuldade da atenção dada aos filhos pelos pais, sendo o estímulo familiar inadequado.

As classes econômicas mais prevalentes na atual pesquisa foram as classes E até C1 (66\%), porém não foram encontradas correlações positivas entre nível socioeconômico e atraso no desenvolvimento. Em relação ao tipo de plano de saúde, $58 \%$ das famílias eram dependentes do Sistema Único de Saúde (SUS), porém este dado não apresentou correlação significativa quanto ao desenvolvimento dos pré-escolares. Acredita-se que como a amostra possuía uma distribuição de renda mais homogênea não foi possível verificar diferenças no impacto desse fator no desenvolvimento das crianças. Tal achado é discordante do estudo de Celikkiran et al. (2015) que avaliaram a prevalência de fatores sociodemográficos relacionados ao desenvolvimento e verificaram que $12 \%$ das crianças avaliadas $(n=1000)$ apresentaram atraso no desenvolvimento com influência do valor da renda familiar mensal.

Em nosso estudo 63\% das mães e 54\% dos pais possuíam ensino médio ou superior completo, não sendo correlacionados com o atraso no desenvolvimento das crianças. Tal achado foi discordante do estudo de Oskan, Senel e Arslan (2012) que avaliaram uma amostra de 692 crianças com idade de três meses a cinco anos e identificaram como fator de risco associado ao pior desempenho no teste de Denver II o baixo nível de escolaridade dos pais.

O aleitamento materno é considerado grande fator de proteção psicossocial relacionado ao crescimento e ao desenvolvimento infantil, além de fornecer à criança o leite como principal fonte de alimento e proteção imunológica, ele possibilita o vínculo mãe-criança. Em nosso estudo, 95\% das crianças foram amamentadas no seio da mãe, porém não se observaram diferenças significativas entre tipo de aleitamento e o risco de atraso no desenvolvimento neuropsicomotor.

A partir dos resultados encontrados pode-se afirmar que os objetivos do estudo foram atingidos. Contudo, algumas limitações do estudo merecem ser mencionadas, tais como o teste de Denver II não ser um teste de diagnóstico do desenvolvimento e o não preenchimento completo de todos os dados referente ao perfil socioeconômico das famílias.

\section{CONCLUSÕES}

Os resultados evidenciaram que a maioria das crianças era saudável em relação aos indicadores biológicos e clínicos, embora fossem classificadas como risco para problemas no desenvolvimento. Foi identificado associação de fatores biológicos (parto normal, altura ao nascimento e altura na idade préescolar) e socioeconômicos (renda familiar mensal) desfavoráveis sobre o desenvolvimento neuropsicomotor dos pré-escolares, sendo a área da linguagem com maior atraso. Por se tratar de uma amostra homogênea, não foram encontradas associações significativas das demais variáveis biológicas e socioeconômicas. A escola constitui importante instutuição que permite aos alunos experiências novas, diferentes das que são experimentadas no ambiente domiciliar, fato que possa ter contribuído no desempenho dos pré-escolares. 


\section{REFERÊNCIAS}

ANDRADE, S. E.; RAEBEL, M. A.; BROWN J.; LANE K.; LIVINGSTON, J.; BOUDREAU, D.; ROLNICK, S. J.; ROBLIN, D.; SMITH, D. H.; WILLY, M. E.; STAFFA, J. A.; PLATT, R. Use of antidepressant medications during pregnancy: a multisite study. Am J Obstet Gynecol., v. 198, n. 2008, p. 194.e1-194.e5.

BALTIERI, L.; SANTOS, D. C. C.; GIBIM, N. C.; SOUZA, C. T.; BATISTELA, A. C. T.; TOLOCKA, R. E. Desempenho motor de lactentes frequentadores de berçários em creches públicas. Rev Paul Pediatr., v. 28, n. 3, 2010, p. 283-289.

BARROS, K. M. F. T.; FRAGOSO, A.G.C.; OLIVEIRA, A.L.B.; CABRAL FILHO, J.E.; CASTRO, R.M. Do environmental influences alter motor abilities acquisition? A comparison among children from day-care centers and private schools. Arq. Neuro-Psiquiatr., v. 61, n. 2A, 2003.

BISCEGLI, T. S.; POLIS. L. B.; SANTOS, L. M.; VICENTIM, M. Avaliação do estado nutricional e do desenvolvimento neuropsicomotor em crianças freqüentadoras de creche. Rev Paul Pediatr, v. 25 n.4, 2007, p. 337-342.

BRITO, C. M. L.; VIEIRA, G. L; COSTA, M. C. O.; OLIVEIRA, N. F. Desenvolvimento neuropsicomotor: o teste de Denver na triagem dos atrasos cognitivos e neuromotores de pré-escolares. Cad Saúde Pública, Rio de Janeiro, v. 27, n. 7, 2011, p.1403-1414.

CAMPOS, A. N.; SILVA, L. H.; PEREIRA, K.; ROCHA, N. A. C. F.; TUDELLA, E. Psychomotor intervention on children of low socioeconomic status. Fisioter Pesqui., São Paulo, v.15, n.2, p.188-93, 2008.

CARVALHO, A. E.V.; LINHARES, M. B. M.; MARTINEZ, F. E. História de desenvolvimento e comportamento de crianças nascidas pré-termo e baixo-peso $(<1500 \mathrm{~g})$. Psicol Reflex e Crít., Porto Alegre, v. 7, n. 1, p. 1-33, 2001.

ÇELIKKIRAN, S.; BOZKURT, H.; COSKUN, M. Denver Developmental Test Findings and their Relationship with Sociodemographic Variables in a Large Community Sample of 0-4-Year-Old Children. Noro Psikiyatr Ars, v. 52, 2015, p.180-184.
Costa, E. F.; CAVAlCANTE, L. I. C.; Dell'aglio, D. D. Language development profile of children in Belen, according to Denver Developmental Screening Test. Rev CEFAC, v. 17, n.4, 2015, p.1090-1102.

CRÓ, M. L.; PINHO, A. M. A primeira infância e a avaliação do desenvolvimento pessoal e social. Rev Ibero-am Educação, Coimbra, n. 56/1, 2011.

CROOKSTON, B. T.; FORSTE, R.; MCCLELLAN, C.; GEORGIADIS, A.; HEATON, T. B. Factors associated with cognitive achievement in late childhood and adolescence: the Young Lives cohort study of children in Ethiopia, India, Peru, and Vietnam. BMC Pediatr, v. 14, n. 253, 2014.

EICKMANN, S. H.; MALKES, N. F. A.; LIMA, M. C. psychomotor development of preterm infants aged 6 to 12 months. São Paulo Med J, v.130, n.5, 2012, p. 299306.

FORMIGA, C. K. M. R. Detecção de risco para problemas no desenvolvimento de bebês nascidos pré-termo no primeiro ano. Tese (Doutorado) -Faculdade de Medicina de Ribeirão Preto, USP, 2009.

FRANKENBURG, W. K.; DODDS, J.; ARCHER, P.; SHAPIRO, H.; BRESNICK, B. The Denver II: A major revision and restandartization of the Denver development screening Test. Pediatrics, Illinois, v. 89, n. 1, 1992, p. 91-97.

YAMAMOTO, R. M.; SCHOEPS, D. O.; ABREU, L. C.; LEONE, C. Peso insuficiente ao nascer e crescimento alcançado na idade pré-escolar, por crianças atendidas em creches filantrópicas do município de Santo André, São Paulo, Brasil. Rev. Bras. Saude Mater. Infant, Recife, v. 9, n.4, 2009, p.477-485.

LOOSLI, N. S.; SOUZA, C. C. S.; UZELOTO, J. S.; OLIVEIRA, E. V.; SANTOS, D. F.; MARCELINO, L. S.; GENARO, S. C.; PACAGNELLI, F. L.; SILVA, R. C. R.; FERNANI, D. C. G. L. Análise de crianças de creche através do Índice de Massa Corporal e Teste de Denver II. Colloquiun Vitae, v. 4, 2012, p.97- 105.

MAGGI, E. F.; MAGALHÃES, L. C.; CAMPOS, A. F.; BOUZADA, M. C. Preterm children have functional performance when compared to term children of 
preschool age. J. Pediatr. (Rio J.), v. 90, n. 4, 2014, p. $377-383$.

MATOS, P. P. Perturbações do desenvolvimento infantilconceitos gerais. Rev Port Clin Geral, Portugal, v.25, n.6, p. 669-676, 2009.

MORAES, M. W.; WEBER, A. P. R.; SANTOS, M. C. O.; ALMEIDA, F. A. Teste de Denver II: avaliação do desenvolvimento de crianças atendidas no ambulatório do Projeto Einstein na Comunidade de Paraisópolis. Einstein, São Paulo, v.8, n.1, p. 149-153, 2010.

MOUSINHO, R.; SCHIMID, E.; PEREIRA, J.; LYRA, L.; MENDES, L.; NÓBREGA, V. Aquisição e desenvolvimento da linguagem: dificuldades que podem surgir neste percurso. Rev Psicopedag., v. 25, n. 78, 2008, p. 297 306.

NAJJAR, E. C. A.; OLIVEIRA, A. I. A.; SILVA, A. P. L; LIMA, A. A. Influence of sociofamilial conditions on the neuropsychomotor development of instituonalized children. Rev Temas Desenvolv., v. 17, n. 100, 2011, p. 155-158.

OZKAN, M.; SENEL, S.; ARSLAN, E. A. The socioeconomic and biological risk factors for developmental delay in early childhood. Eur J Pediatr, v. 171, 2012, p. 18151821.

PILZ,E.M.L.; SCHERMANN, L. B. Determinantes biológicos e ambientais no desenvolvimento neuropsicomotor em uma amostra de crianças de Canoas/RS. Ciênc Saúde Colet., Rio Grande do Sul, v. 12, n. 1, 2007, p.181-190.

PEREIRA, A. S.; PEIXOTO, N. G. A.; NOGUEIRA NETO, J. F.; LANZILLOTTI, H. S.; SOARES, E. A. Estado nutricional de pré-escolares de creche pública: um estudo longitudinal. Cad Saúde Coletiva, Rio de Janeiro, v.21, n.2, 2013, p.140-147.

PULIDO, J. H. P.; BARRETO, L. C. R.; TORRES, J. D. C. Relación entre peso al nacer y madurezneuropsicológica enpreescolares de Tunja (Colômbia). Pensam. Psicol., v. 13, n. 2, 2015, p. 65-77.

RONFANI, L.; VECCHI, B. L.; MARIUZ, M.; TOGNIN, V.; BIN, M.; FERLUGA, V.; KNOWLES, A.; MONTICO, M.;
BARBONE, F. The Complex Interaction between Home Environment, Socioeconomic Status, Maternal IQ and early child neurocognitive development: a multivariate analysis of data collected in a newborn cohort study. PlosOne, v. 10, n.5, 2015.

SILVA, A. C. D.; ENGSTRON, E. M.; MIRANDA, C. T. Fatores associados ao desenvolvimento neuropsicomotor em crianças de 6-18 meses de vida inseridas em creches públicas do Município de João Pessoa, Paraíba, Brasil. Cad Saúde Pública, Rio de Janeiro, v. 31, n. 9, 2015, p. 1881-1893.

SOUZA, S. C.; LEONE, C.; TAKANO, O. L.; MORATELLI, H. B. Desenvolvimento de pré-escolares na educação infantil em Cuiabá, Mato Grosso, Brasil, Cad Saúde Pública, Rio de Janeiro, v. 24, n. 8, 2008, p. 1917-1926.

TIAGO, D. A.; SANCHES, K. C.; SÁ, C. S. C. Avaliação do desenvolvimento neuropsicomotor de crianças em período pré-escolar. Rev Neurociênc., v.23, n.3, 2015, p. 413-419.

TORQUATO,J.A.; PAES, J. B.; BENTO, M.C.C.; SAIKAI, G. M. P. P.; SOUTO, J. N.; LIMA, E. A. M.; ABREU, L. C. Prevalência de atraso do desenvolvimento neuropsicomotor em préescolares. Rev Bras Crescimento e Desenvolv Hum., v.21, n.9, 2011, p. 259-268.

VERISSÍMO, M.; FERNANDES, C.; SANTOS, A. J.; PECEGUINA, I.; VAUGHN, B. E.; BOST, K. K. The Relation between Quality of Attachment and the Development of Social Competence in Preschool Children. Psicol Reflex e Crít, v.2, n.2, 2011, p.292-299.

Recebido em: 14 de outubro de 2016 Aceito em: 27 de março de 2017 